\title{
Responses to applied forces and the Jarzynski equality in classical oscillator systems coupled to finite baths: An exactly solvable non-dissipative non-ergodic model
}

\author{
Hideo Hasegawa* \\ Department of Physics, Tokyo Gakugei University, Koganei, Tokyo 184-8501, Japan
}

(Dated: November 6, 2018)

\begin{abstract}
Responses of small open oscillator systems to applied external forces have been studied with the use of an exactly solvable classical Caldeira-Leggett (CL) model in which a harmonic oscillator (system) is coupled to finite $N$-body oscillators (bath) with an identical frequency $\left(\omega_{n}=\omega_{o}\right.$ for $n=1$ to $N$ ). We have derived exact expressions for positions, momenta and energy of the system in nonequilibrium states and for work performed by applied forces. Detailed study has been made on an analytical method for canonical averages of physical quantities over the initial equilibrium state, which is much superior than numerical averages commonly adopted in simulations of small systems. The calculated energy of the system which is strongly coupled to finite bath is fluctuating but non-dissipative. It has been shown that the Jarzynski equality (JE) is valid in non-dissipative, non-ergodic open oscillator systems regardless of the rate of applied ramp force.

PACS numbers: 05.70.-a, 05.40.-a, 05.10.Gg
\end{abstract}

*hideohasegawa@goo.jp 


\section{INTRODUCTION}

In the last decade, a significant progress has been made in our understanding of nonequilibrium statistics. Experimental and theoretical studies have been developed on small systems such as quantum dots and biological molecular machines which generally operate away from equilibrium (for reviews, see Refs. [1 3 ] $]$ ). The development of modern techniques of microscopic manipulation has promoted experimental studies of small systems. It has become possible to study the response of small systems to applied external forces. In parallel theorists have developed the important three theorems: the Jarzynski equality (JE) [4], the steady-state and transient fluctuation theorems [5] 7], and Crook's theorem [6, 7]. These fluctuation theorems may be applicable to nonequilibrium systems driven far from the equilibrium states. In this paper we pay our attention to a remarkable JE given by

$$
e^{-\beta \Delta F}=\left\langle e^{-\beta W}\right\rangle=\int d W P(W) e^{-\beta W}
$$

where $W$ denotes a work made in a system when its parameter is changed, the bracket $\langle\cdot\rangle$ expresses the average over the work distribution function (WDF) $P(W)$ of work performed by a prescribed protocol, $\Delta F$ stands for the free energy difference between the initial and final equilibrium states, and $\beta\left(=1 / k_{B} T\right)$ is the inverse temperature of the initial state. Equation (1) includes the second law of thermodynamics: $\langle W\rangle \geq \Delta F$, where the equality holds only for the reversible process. The JE was originally proposed for classical isolated system and open system weakly coupled to baths which are described by the Hamiltonian [4] and the stochastic models [8]. Jarzynski later proved that the JE is valid for strongly coupled open systems [9]. A generalization of the JE to quantum systems has been made in Refs. [10]-[17].

A validity of the JE has been confirmed by some experiments [18 23]. Liphardt et. al. [18] have determined the free energy required to unfold a single RNA chain from nonequilibrium work measurements. Wang et. al. [19] have considered a colloidal particle pulled through liquid water by an optical trap. Douarche et. al. [20, 21] have verified the JE for a mechanical oscillator that is driven out of equilibrium by an external force. By using a torsion pendulum composed of a brass wire, Joubaud et. al., [22, 23] have experimentally studied the JE of the harmonic oscillator in contact with a thermostat and driven out of equilibrium by an external force. 
Some criticisms, however, have been raised for the validity of the JE [24]-[35]. Cohen and Mauzerall [24] pointed out that it is difficult to define the distribution and the temperature during the irreversible process. In response to this criticism, Jarzynski [9] has claimed that the JE holds if the initial state is in the equilibrium state with the definite temperature [9]. It has been pointed out that the JE may be violated in ideal gas model [25, 31, 32, 34, 35] and in a rigid rotator model [26, 28, 29]. Therefore it is currently an important issue to examine the validity condition of the JE.

Many studies have been reported for harmonic oscillator systems by both experimental [3, 18, 20 23] and theoretical methods [36]-[46]. Theoretical analyses have been made for oscillators with the use of the Markovian Langevin model [20 23, 36], the non-Markovian Langevin model [37-40], Fokker-Planck equation [41], and Hamiltonian model [42 46]. All of these studies have shown that the JE holds in isolated and open oscillators, assuming dissipative memory kernels or the over-damped models. This assumption seems reasonable in the situation under which the relevant experiments [20 23] have been performed. Recent theoretical studies, however, have demonstrated that the energy dissipation is not realized in a small system coupled to finite thermal baths [47, 48]. This is quite different from the case of infinite baths in which dissipation is realized. Indeed, it is commonly believed that the dissipation is realized only when the system is coupled to infinite bath (except for chaotic baths) [49]. Poicaré recurrence time is finite for finite bath.

It is necessary to make detailed calculations of responses of small systems to the applied force such as variations of position and energy of the system, which have not been reported as far as we are ware of. The purpose of the present study is twofold: to make detailed study of the response to an applied force and to examine the validity of the JE in open harmonic oscillator systems in the non-dissipative situation. We consider the CaldeiraLeggett (CL) Hamiltonian model [50, 51], adopting a single- $\omega$ bath containing uncoupled $N$-body oscillators with an identical frequency: $\omega_{n}=\omega_{o}$ for $n=1$ to $N$ [Eq. (13)]. The CL model with a single- $\omega$ bath is exactly solvable. A similar optic-phonon-mode model for bath was adopted in a different context from the present study [52]. In the conventional approach, we obtain the Langevin equation from the CL model, with which its properties are investigated. In this study, we have directly obtained the Laplace-transformed equation of motion of the system. The energy and work of the system induced by the applied force are analytically averaged over the canonical distribution of initial equilibrium states. Our non- 
dissipative system-plus-bath yields non-ergodic solutions, for which the JE will be shown to be valid in contrast with Refs. [39, 40] claiming the importance of the ergodicity.

The paper is organized as follows. In the next Sec. II, we derive expressions of response of positions, momenta and system energy induced by an applied ramp force in open oscillator systems, by using the CL model with the single- $\omega$ bath mentioned above. We obtain the WDF and the averaged work with which the validity of the JE have been investigated. Some numerical calculations are presented. In Sec. III an application of other types of external forces to the system is studied. We compare our study with the method using the Langevin model derived from the CL model. Sec. IV is devoted to our conclusion.

\section{THE ADOPTED MODEL}

\section{A. Equations of motion}

We consider a system of a classical oscillator coupled to a bath consisting of $N$-body uncoupled oscillators described by the CL model [50, 51],

$$
H=H_{S}+H_{B}+H_{I}
$$

with

$$
\begin{aligned}
& H_{S}=\frac{P^{2}}{2 M}+\frac{M \Omega^{2} Q^{2}}{2}-f(t) Q \\
& H_{B}=\sum_{n=1}^{N}\left(\frac{p_{n}^{2}}{2 m}+\frac{m \omega_{n}^{2} q_{n}^{2}}{2}\right) \\
& H_{I}=-\sum_{n=1}^{N}\left(c_{n} q_{n} Q-\frac{c_{n}^{2} Q^{2}}{2 m \omega_{n}^{2}}\right)
\end{aligned}
$$

where $H_{S}, H_{B}$ and $H_{I}$ express one-dimensional Hamiltonians of the system, bath and interaction, respectively, $M(m), \Omega\left(\omega_{n}\right), Q\left(q_{n}\right)$ and $P\left(p_{n}\right)$ denote mass, frequency, position and momentum, respectively, of the system (bath), $c_{n}$ the interaction between the system and bath, and $f(t)$ an applied external force. Equations of motion for $Q$ and $q_{n}$ are given by

$$
\begin{aligned}
& M \ddot{Q}=-M \Omega^{2} Q+\sum_{n=1}^{N} c_{n}\left(q_{n}-\frac{c_{n} Q}{m \omega_{n}^{2}}\right)+f(t), \\
& m \ddot{q}_{n}=-m \omega_{n}^{2} q_{n}+c_{n} Q .
\end{aligned}
$$


Applying the Laplace transformation to Eqs. (66) and (7), we obtain

$$
\begin{aligned}
& M\left[s^{2} \hat{Q}(s)-\dot{Q}(0)-s Q(0)\right]=-M \Omega^{2} \hat{Q}(s)-\sum_{n=1}^{N}\left(\frac{c_{n}^{2}}{m \omega_{n}^{2}}\right) \hat{Q}(s)+\sum_{n=1}^{N} c_{n} \hat{q}_{n}(s)+\hat{f}(s), \\
& m\left[s^{2} \hat{q}_{n}(s)-\dot{q}_{n}(0)-s q_{n}(0)\right]=-m \omega_{n}^{2} \hat{q}_{n}(s)+c_{n} \hat{Q}(s),
\end{aligned}
$$

where

$$
\hat{Q}(s)=\int_{0}^{\infty} d t e^{-s t} Q(t)
$$

and similar expressions for $\hat{q}_{n}(s)$ and $\hat{f}(s)$. Solving Eq. (9) in terms of $\hat{q}(s)$ and substituting it into Eq. (8), we obtain

$$
\hat{Q}(s)=\hat{G}(s)\left[\dot{Q}(0)+s Q(0)+\sum_{n=1}^{N} \frac{c_{n}\left[\dot{q}_{n}(0)+s q_{n}(0)\right]}{M\left(s^{2}+\omega_{n}^{2}\right)}+\frac{\hat{f}(s)}{M}\right],
$$

where the Green's function $\hat{G}(s)$ is given by

$$
\hat{G}(s)=\left(s^{2}+\Omega^{2}+\sum_{n=1}^{N} \frac{c_{n}^{2} s^{2}}{M m \omega_{n}^{2}\left(s^{2}+\omega_{n}^{2}\right)}\right)^{-1} .
$$

In order to make analytic calculation feasible, we consider a bath containing $N$-body uncoupled oscillators with an identical frequency $\omega_{o}$ and a uniform coupling $c_{o}$, as given by

$$
\begin{aligned}
& \omega_{n}=\omega_{o}, \\
& c_{n}=\frac{c_{o}}{\sqrt{N}} \quad \text { for } n=1 \text { to } N .
\end{aligned}
$$

We have chosen $c_{n}$ such that it yields a non-divergent result in the limit of $N \rightarrow \infty$ in Eq. (12) (related discussion being given in Sec. III.B) [53]. With the use of Eqs. (13) and (14), $\hat{Q}(s)$ becomes

$$
\hat{Q}(s)=\hat{G}(s)\left[\frac{P_{0}}{M}+s Q_{0}+\frac{c_{o}}{M \sqrt{N}\left(s^{2}+\omega_{o}^{2}\right)} \sum_{n=1}^{N}\left(\frac{p_{n 0}}{m}+s q_{n 0}\right)+\frac{\hat{f}(s)}{M}\right],
$$

with

$$
\hat{G}(s)=\left(s^{2}+\Omega^{2}+\frac{c_{o}^{2} s^{2}}{M m \omega_{o}^{2}\left(s^{2}+\omega_{o}^{2}\right)}\right)^{-1},
$$

where $P_{0}=M \dot{Q}(0), Q_{0}=Q(0), p_{n 0}=m \dot{q}_{n}(0)$ and $q_{n 0}=q_{n}(0)$. Equation (16) may be rewritten as

$$
\begin{aligned}
\hat{G}(s) & =\frac{s^{2}+\omega_{o}^{2}}{\left[\left(s^{2}+\Omega^{2}\right)\left(s^{2}+\omega_{o}^{2}\right)+c_{o}^{2} s^{2} / M m \omega_{o}^{2}\right]}, \\
& =\sum_{i=1}^{2} \frac{b_{i}}{\left(s^{2}+a_{i}^{2}\right)}
\end{aligned}
$$


with

$$
\begin{aligned}
a_{i}^{2} & =\frac{1}{2}\left[\Omega^{2}+\omega_{o}^{2}+\frac{c^{2}}{M m \omega_{o}^{2}}+(-1)^{i-1} \sqrt{D_{o}}\right] \quad(i=1,2), \\
D_{o} & =\left(\Omega^{2}-\omega_{o}^{2}\right)^{2}+\frac{2 c_{o}^{2}\left(\Omega^{2}+\omega_{o}^{2}\right)}{M m \omega_{o}^{2}}+\frac{c_{o}^{4}}{M^{2} m^{2} \omega_{o}^{4}} \geq 0, \\
b_{1} & =\frac{a_{1}^{2}-\omega_{o}^{2}}{a_{1}^{2}-a_{2}^{2}}, \quad b_{2}=\frac{\omega_{o}^{2}-a_{2}^{2}}{a_{1}^{2}-a_{2}^{2}} .
\end{aligned}
$$

Then Eq. (15) becomes

$$
\hat{Q}(s)=\sum_{i=1}^{2} \frac{b_{i}}{\left(s^{2}+a_{i}^{2}\right)}\left[\frac{P_{0}}{M}+s Q_{0}+\frac{c_{o}}{M \sqrt{N}\left(s^{2}+\omega_{o}^{2}\right)} \sum_{n=1}^{N}\left(\frac{p_{n 0}}{m}+s q_{n 0}\right)+\frac{\hat{f}(s)}{M}\right],
$$

whose inverse Laplace transformation yields

$$
Q(t)=\Phi(t)+X_{Q}(t) Q_{0}+X_{P}(t) P_{0}+Y_{q}(t) \sum_{n=1}^{N} q_{n 0}+Y_{p}(t) \sum_{n=1}^{N} p_{n 0}
$$

with

$$
\begin{aligned}
\Phi(t) & =\sum_{i=1}^{2} \frac{b_{i}}{M a_{i}} \int_{0}^{t} \sin a_{i}\left(t-t^{\prime}\right) f\left(t^{\prime}\right) d t^{\prime}, \\
X_{Q}(t) & =\sum_{i=1}^{2} b_{i} \cos a_{i} t, \\
X_{P}(t) & =\sum_{i=1}^{2}\left(\frac{b_{i}}{M a_{i}}\right) \sin a_{i} t, \\
Y_{q}(t) & =\sum_{i=1}^{2}\left(\frac{b_{i} c_{o}}{M \sqrt{N}}\right) \frac{\left(\cos \omega_{o} t-\cos a_{i} t\right)}{\left(a_{i}^{2}-\omega_{o}^{2}\right)}, \\
Y_{p}(t) & =\sum_{i=1}^{2}\left(\frac{b_{i} c_{o}}{M \sqrt{N}}\right) \frac{\left(a_{i} \sin \omega_{o} t-\omega_{o} \sin a_{i} t\right)}{m \omega_{o} a_{i}\left(a_{i}^{2}-\omega_{o}^{2}\right)} .
\end{aligned}
$$

\section{B. Position, momentum and system energy}

It is necessary to evaluate physical quantities averaged over the canonical distribution of initial states, $Q_{0}, P_{0},\left\{q_{n 0}\right\}$ and $\left\{p_{n 0}\right\}$, of the equilibrium coupled system-and-bath $H(t=0)$. In order to make such evaluations, we need following (fluctuation-dissipation) relations for 
$f(0)=0$ given by

$$
\begin{aligned}
M \Omega^{2}\left\langle Q_{0}^{2}\right\rangle_{0} & =\frac{\left\langle P_{0}^{2}\right\rangle_{0}}{M}=k_{B} T=\frac{1}{\beta} \\
m \omega_{o}^{2}\left\langle q_{n 0} q_{\ell 0}\right\rangle_{0} & =k_{B} T \delta_{n \ell}+\frac{c_{n} c_{\ell} k_{B} T}{m \omega_{o}^{2} M \Omega^{2}} \\
\frac{\left\langle p_{n 0} p_{\ell 0}\right\rangle_{0}}{m} & =k_{B} T \delta_{n \ell} \\
\left\langle Q_{0} q_{n 0}\right\rangle_{0} & =\frac{c_{n} k_{B} T}{m \omega_{0}^{2} M \Omega^{2}} \\
\left\langle P_{0} Q_{0}\right\rangle_{0} & =\left\langle P_{0} q_{n 0}\right\rangle_{0}=\left\langle P_{0} p_{n 0}\right\rangle_{0}=\left\langle p_{n 0} q_{\ell 0}\right\rangle_{0}=\left\langle p_{n 0} Q\right\rangle_{0}=0 \\
\left\langle Q_{0}\right\rangle_{0} & =\left\langle P_{0}\right\rangle_{0}=\left\langle q_{n 0}\right\rangle_{0}=\left\langle p_{n 0}\right\rangle_{0}=0
\end{aligned}
$$

with

$$
\langle O\rangle_{0} \equiv \frac{\operatorname{Tr}\left\{e^{-\beta H(0)} O\right\}}{\operatorname{Tr} e^{-\beta H(0)}}
$$

where $O$ denotes an operator and $\operatorname{Tr}$ the trace over initial state of $H(0)$ with $Q_{0}, P_{0},\left\{q_{n 0}\right\}$ and $\left\{p_{n 0}\right\}$. Equations (30) and (32) arise from the relation,

$$
m \omega_{o}^{2}\left\langle\left(q_{n 0}-\frac{c_{n} Q_{0}}{m \omega_{o}^{2}}\right)\left(q_{\ell 0}-\frac{c_{\ell} Q_{0}}{m \omega_{o}^{2}}\right)\right\rangle_{0}=k_{B} T \delta_{n \ell}
$$

In the limit of $c_{o}=0$, Eqs. (29)-(34) reduce to the well-known result for isolated system and bath. With the use of Eqs. (23) and (34), the averaged position and momentum of the system are given by

$$
\begin{aligned}
& \bar{Q}(t)=\langle Q(t)\rangle_{0}=\Phi(t)=\int_{0}^{t} \chi\left(t-t^{\prime}\right) f\left(t^{\prime}\right) d t^{\prime} \\
& \bar{P}(t)=\langle P(t)\rangle_{0}=M \dot{\Phi}(t)=M \int_{0}^{t} \dot{\chi}\left(t-t^{\prime}\right) f\left(t^{\prime}\right) d t^{\prime},
\end{aligned}
$$

with the time-dependent susceptibility $\chi(t)$,

$$
\chi(t)=\sum_{i=1}^{2} \frac{b_{i} \sin a_{i} t}{M a_{i}},
$$

where dot $(\cdot)$ stands for the derivative with respect to time. It is easy to see from Eq. (15) that the Laplace-transformed susceptibility is given by $\hat{\chi}(s)=\hat{G}(s) / M$. The frequencydependent susceptibility $\chi(\omega)$ is given by

$$
\begin{aligned}
\chi(\omega) & =\int_{0}^{\infty} e^{-i \omega t} \chi(t) d t=\hat{\chi}(-i \omega), \\
& =-\frac{1}{M} \sum_{i=1}^{2} \frac{b_{i}}{a_{i}\left(\omega^{2}-a_{i}^{2}\right)},
\end{aligned}
$$


whose imaginary part becomes

$$
\operatorname{Im} \chi(\omega)=\sum_{i=1}^{2}\left(\frac{\pi M b_{i}}{2 a_{i}}\right)\left[\delta\left(\omega-a_{i}\right)-\delta\left(\omega+a_{i}\right)\right] .
$$

The system energy $\bar{E}_{S}$ averaged over the initial state is given by $[54,57]$

$$
\bar{E}_{S}=\left\langle E_{S}\right\rangle_{0}=\frac{M}{2}\left\langle\dot{Q}^{2}\right\rangle_{0}+\frac{M \Omega^{2}}{2}\left\langle Q^{2}\right\rangle_{0}-f(t)\langle Q\rangle_{0} .
$$

By using (29)-(34) and Eqs. (43), we obtain $\bar{E}_{S}$ given by

$$
\bar{E}_{S}=\bar{E}_{S}^{(0)}+\bar{E}_{S}^{(f)}
$$

with

$$
\begin{aligned}
\bar{E}_{S}^{(0)} & =\frac{k_{B} T}{2 M \Omega^{2}}\left[M \dot{X}_{Q}(t)^{2}+M \Omega^{2} X_{Q}(t)^{2}\right] \\
& +\frac{M k_{B} T}{2}\left[M \dot{X}_{P}(t)^{2}+M \Omega^{2} X_{P}(t)^{2}\right]+\frac{N m k_{B} T}{2}\left[M \dot{Y}_{p}(t)^{2}+M \Omega^{2} Y_{p}(t)^{2}\right] \\
& +\frac{N k_{B} T}{2 m \omega^{2}}\left(1+\frac{c_{o}^{2}}{m \omega_{o}^{2} M \Omega^{2}}\right)\left[M \dot{Y}_{q}(t)^{2}+M \Omega^{2} Y_{q}(t)^{2}\right] \\
& +\frac{\sqrt{N} c_{o} k_{B} T}{m \omega_{o}^{2} M \Omega^{2}}\left[M \dot{X}_{Q}(t) \dot{Y}_{q}(t)+M \Omega^{2} X_{Q}(t) Y_{q}(t)\right] \\
\bar{E}_{S}^{(f)} & =\frac{1}{2}\left[M \dot{\Phi}(t)^{2}+M \Omega^{2} \Phi(t)^{2}\right]-f(t) \Phi(t) .
\end{aligned}
$$

Here $\bar{E}_{S}^{(0)}$ expresses the system energy depending on the temperature but independent of the applied force: $\bar{E}_{S}^{(f)}$ denotes the response to the force: $\Phi(t), X_{Q}(t), X_{P}(t), Y_{q}(t)$ and $Y_{p}(t)$ are given by Eqs. (24)-(28) $: \dot{X}_{Q}(t), \dot{X}_{P}(t), \dot{Y}_{q}(t)$ and $\dot{Y}_{p}(t)$ are their derivatives with respect to time. It is noted that $\bar{Q}(t)$ and $\bar{P}(t)$ are independent of $N$ because of the $N$-independent $\Phi(t)$ in Eq. (24). Furthermore $\bar{E}_{S}$ does not depend on $N$ because the $N$ factor in the fourth term of Eq. (45) is canceled out by the $1 / N$ term in $Y_{q}(t)^{2}$ in Eq. (27) and because the $\sqrt{N}$ term of the last term of Eq. (45) is cancelled out by the $1 / \sqrt{N}$ of $Y_{q}(t)$. These properties arise from our adopted model with $c_{n}=c_{o} / \sqrt{N}$ in Eq. (14) [53].

The advantage of expressions given by Eqs. (37), (38) and (44)-(46) is that canonical averages over the initial state have been analytically made and they are free from the numerical averaging which is one of difficulties in direct simulations of small systems [47, 48, 58 60].

We have so far not specified the form of an external force $f(t)$. For a while we consider 
a ramp force given by

$$
f(t)= \begin{cases}0 & \text { for } t<0, \\ g\left(\frac{t}{\tau}\right) & \text { for } 0 \leq t<\tau, \\ g & \text { for } t \geq \tau,\end{cases}
$$

where $\tau$ stands for a duration of the applied force and $g$ the magnitude of the force at $t \geq \tau$. For the ramp force, Eq. (24) leads to

$$
\begin{aligned}
\Phi(t) & =\sum_{i=1}^{2}\left(\frac{g b_{i}}{M a_{i}^{3} \tau}\right)\left(a_{i} t-\sin a_{i} t\right) \quad \text { for } 0 \leq t<\tau, \\
& =\sum_{i=1}^{2}\left(\frac{g b_{i}}{M a_{i}^{2}}\right)\left(\frac{1}{a_{i} \tau}\left[a_{i} \tau+\sin a_{i}(t-\tau)-\sin a_{i} t\right]\right) \quad \text { for } t \geq \tau .
\end{aligned}
$$

In the following, we examine the three cases of (1) no couplings $\left(c_{o}=0\right),(2)$ transient force $(\tau=0)$ and $(3)$ quasi-static force $(\tau \rightarrow \infty)$.

(1) In the case of $c_{o}=0$ where Eqs. (19)-(21) lead to $a_{1}=\Omega, a_{2}=\omega_{o}, b_{1}=1$ and $b_{2}=0$, we obtain

$$
\begin{aligned}
\Phi(t) & =\left(\frac{g}{M \Omega^{3} \tau}\right)(\Omega t-\sin \Omega t) \quad \text { for } 0 \leq t<\tau, \\
& =\left(\frac{g}{M \Omega^{2}}\right)\left(\frac{1}{\Omega \tau}[\Omega \tau+\sin \Omega(t-\tau)-\sin \Omega t]\right) \quad \text { for } t \geq \tau .
\end{aligned}
$$

Equations (25) and (26) lead to

$$
\begin{aligned}
& X_{Q}(t)=\cos \Omega t \\
& X_{P}(t)=\left(\frac{1}{M \Omega}\right) \sin \Omega t
\end{aligned}
$$

$\bar{E}_{S}(t)$ becomes

$$
\begin{aligned}
\bar{E}_{S}(t) & =k_{B} T-\frac{g^{2}}{2 M \Omega^{2}}\left[\left(\frac{t}{\tau}\right)^{2}-\frac{2(1-\cos \Omega t)}{\Omega^{2} \tau^{2}}\right] \quad \text { for } 0 \leq t<\tau, \\
& =k_{B} T-\frac{g^{2}}{2 M \Omega^{2}}\left[1-\frac{2(1-\cos \Omega \tau)}{\Omega^{2} \tau^{2}}\right] \quad \text { for } t \geq \tau .
\end{aligned}
$$

(2) In the case of $\tau=0$, Eq. (49) yields

$$
\Phi(t)=\sum_{i=1}^{2}\left(\frac{g b_{i}}{M a_{i}^{2}}\right)\left(1-\cos a_{i} t\right) \quad \text { for } t \geq 0
$$


which becomes for $c_{o}=0$,

$$
\Phi(t)=\left(\frac{g}{M \Omega^{2}}\right)(1-\cos \Omega t) \quad \text { for } t \geq 0,
$$

yielding

$$
\bar{E}_{S}(t)=k_{B} T \quad \text { for } t \geq 0
$$

(3) In the case of $\tau \rightarrow \infty$, Eq. (48) yields

$$
\Phi(t)=\sum_{i=1}^{2} \frac{g b_{i}}{M a_{i}^{2}}\left(\frac{t}{\tau}\right) \quad \text { for } 0 \leq t<\infty
$$

which becomes for $c_{o}=0$,

$$
\Phi(t)=\frac{g}{M \Omega^{2}}\left(\frac{t}{\tau}\right) \quad \text { for } 0 \leq t<\infty,
$$

leading to

$$
\begin{aligned}
\bar{E}_{S}(t) & =k_{B} T-\frac{g^{2}}{2 M \Omega^{2}}\left(\frac{t}{\tau}\right)^{2}, \\
& =k_{B} T-\frac{g^{2}}{2 M \Omega^{2}} \quad \text { for } t=\tau \rightarrow \infty .
\end{aligned}
$$

We have performed numerical calculations for averaged position, momentum and energy of the system with $M=m=1.0, \Omega=\omega_{o}=1.0$ and $g=1.0$ which are adopted in all our calculations otherwise noticed. Position, momentum and energy (work) are measured in units of $\sqrt{k_{B} T / M \Omega^{2}}, \sqrt{M k_{B} T}$ and $k_{B} T$, respectively. Model calculations of averaged positions and momenta are presented in Figs. 1(a)-(h) where solid and dashed curves express $\bar{Q}(t)$ and $\bar{P}(t)$, respectively. Figures 1 (a) and (b) show the results of $c_{o}=0.0$ and $c_{o}=1.0$, respectively, when a ramp force with $\tau=100$ is applied. Figure 1)(a) shows that $\bar{Q}(t)$ is linearly increased at $0 \leq t<100.0$, and it becomes constant at $t \geq 100.0$ where a force $g$ is still applied. This behavior is not changed even when the system-bath coupling is introduced as shown in Fig. 11(b). Figures 1(c), (e) and (g) show $\bar{Q}(t)$ and $\bar{P}(t)$ for ramp forces with $\tau=10.0,5.0$ and 0.0, respectively, applied to uncoupled systems $\left(c_{o}=0.0\right)$, where regular oscillations are induced. Figures 1(d), (f) and (h), however, show that irregular oscillations are induced by external forces with $\tau=10.0,5.0$ and 0.0 in coupled systems.

Model calculations of system energy $\bar{E}_{S}(t)$ are plotted in Figs. 2(a)-(j). Figures 2(a) and (b) show $\bar{E}_{S}(t)$ for $c_{o}=0.0$ and $c_{o}=1.0$, respectively, without external forces $[f(t)=g=0.0]$ 
for which $\bar{E}_{S}$ is constant. Figures 2(c) and (e) (Figs. 2(d) and (f)) show $\bar{E}_{S}$ for $c_{o}=0.0$ $\left(c_{o}=1.0\right)$, with applied forces of $\tau=100.0$ and 10.0, respectively, where $\bar{E}_{S}$ is gradually decreased by an applied force. As far as the uncoupled system is concerned, this behavior is not modified when the force with smaller $\tau$ is applied, as shown by Figs. 2(g) and (i) for $\tau=5.0$ and $\tau=0.0$, respectively. However, when the ramp force with smaller $\tau$ is applied to coupled systems, the behavior is changed: irregular oscillations are induced in $\bar{E}_{S}$ as shown by Fig. $2(\mathrm{~h})$ and (j) for $\tau=5.0$ and $\tau=0.0$, respectively. These oscillations in coupled systems are realized for ramp forces with $\tau \lesssim T_{o}$ where $T_{o}(=2 \pi / \Omega)$ denotes the period of system oscillation. We note in Figs. $2(\mathrm{~h})$ and $(\mathrm{j})$ that this irregular oscillation is not dissipate, which has been confirmed with calculations for $t \in[0,10000]$ (relevant results not shown). The averaged system energy in the coupled small systems shows irregular non-dissipative oscillations although the total energy of the system-plus-bath is constant [47, 48].

\section{Work and work distribution function}

Next we consider a work performed by an applied external force. By using $Q(t)$ given by Eq. (23), we obtain the work performed by the force $f(t)$ applied for $0 \leq t<\tau$ [4],

$$
\begin{aligned}
W_{0} & =-\int_{0}^{\tau} d t \dot{f}(t) Q(t), \\
& =\phi+C_{Q} Q_{0}+C_{P} P_{0}+D_{q} \sum_{n=1}^{N} q_{n 0}+D_{p} \sum_{n=1}^{N} p_{n 0},
\end{aligned}
$$

where

$$
\begin{aligned}
\phi & =-\int_{0}^{\tau} d t \dot{f}(t) \Phi(t), \\
C_{\xi} & =-\int_{0}^{\tau} d t \dot{f}(t) X_{\xi}(t) \quad(\text { for } \xi=Q \text { and } P), \\
D_{\eta} & =-\int_{0}^{\tau} d t \dot{f}(t) Y_{\eta}(t) \quad(\text { for } \eta=q \text { and } p) .
\end{aligned}
$$

With the use of Eqs. (64)-(67), the WDF of $P(W)$ is given by

$$
\begin{aligned}
P(W) & =\left\langle\delta\left(W-W_{0}\right)\right\rangle_{0}, \\
& =\frac{1}{2 \pi} \int d u \exp (i u W)\left\langle\exp \left(-i u W_{0}\right)\right\rangle_{0},
\end{aligned}
$$


where

$$
\begin{aligned}
\left\langle\exp \left(-i u W_{0}\right)\right\rangle_{0} & =\exp (-i u \phi)\left(\frac{\beta \Omega}{2 \pi}\right)\left(\frac{\beta \omega_{0}}{2 \pi}\right)^{N} \int d Q_{0} \exp \left[-\frac{\beta M \Omega^{2} Q_{0}^{2}}{2}-i u C_{Q} Q_{0}\right] \\
& \times \int d P_{0} \exp \left[-\frac{\beta P_{0}^{2}}{2 M}-i u C_{P} P_{0}\right] \\
& \times \prod_{n=1}^{N} \int d q_{n 0} \exp \left[-\frac{\beta m \omega_{n}^{2}}{2}\left(q_{n 0}-\frac{c_{n} Q_{0}}{m \omega_{o}^{2}}\right)^{2}-i u D_{q}\left(q_{n 0}-\frac{c_{n} Q_{0}}{m \omega_{o}^{2}}\right)-\frac{i u D_{q} c_{n} Q_{0}}{m \omega_{o}^{2}}\right] \\
& \times \prod_{n=1}^{N} \int d p_{n 0} \exp \left(-\frac{\beta p_{n 0}^{2}}{2 m}-i u D_{p} p_{n 0}\right) .
\end{aligned}
$$

Performing the Gauss integrals, we obtain

$$
\left\langle\exp \left(-i u W_{0}\right)\right\rangle_{0}=\exp \left[-i u \phi-\frac{u^{2}}{2 \sigma^{2}}\right]
$$

where

$$
\sigma^{2}=\frac{1}{\beta}\left[\frac{1}{M \Omega^{2}}\left(C_{Q}+\frac{\sqrt{N} c_{o} D_{q}}{m \omega_{o}^{2}}\right)^{2}+M C_{P}^{2}+\frac{N D_{q}^{2}}{m \omega_{o}^{2}}+m N D_{p}^{2}\right]
$$

With the use of Eqs. (69) and (71), $P(W)$ is finally given by

$$
P(W)=\frac{1}{\sqrt{2 \pi \sigma^{2}}} \exp \left[-\frac{(W-\mu)^{2}}{2 \sigma^{2}}\right]
$$

with

$$
\mu=\langle W\rangle=\phi
$$

where $\phi$ and $\sigma^{2}$ are given by Eqs. (65) and (72), respectively. The average of $e^{-\beta W}$ over $P(W)$ is given by

$$
\left\langle e^{-\beta W}\right\rangle=\int d W P(W) e^{-\beta W}=e^{-\beta\left(\mu-\beta \sigma^{2} / 2\right)}
$$

which leads to

$$
\begin{aligned}
R & \equiv-\frac{1}{\beta} \ln \left\langle e^{-\beta W}\right\rangle, \\
& =\mu-\frac{\beta \sigma^{2}}{2}=\phi-\frac{\beta \sigma^{2}}{2} .
\end{aligned}
$$

It is worthwhile to point out that Eq. (75) may be alternatively obtainable by

$$
\left\langle e^{-\beta W_{0}}\right\rangle_{0}=e^{-\beta\left(\phi-\beta \sigma^{2} / 2\right)},
$$


where $\phi$ and $\sigma^{2}$ are given by Eqs. (65) and (72), respectively.

For a ramp force given by Eq. (47), Eqs. (65)-(67) are given by

$$
\begin{aligned}
\phi & =-\left(\frac{g^{2}}{M}\right) \sum_{i=1}^{2} b_{i}\left[\frac{1}{2 a_{i}^{2}}-\frac{\left(1-\cos a_{i} \tau\right)}{a_{i}^{4} \tau^{2}}\right] \\
C_{Q} & =-g \sum_{i=1}^{2} \frac{b_{i} \sin a_{i} \tau}{a_{i} \tau} \\
C_{P} & =-\left(\frac{g}{M}\right) \sum_{i=1}^{2} \frac{b_{i}\left(1-\cos a_{i} \tau\right)}{a_{i}^{2} \tau} \\
D_{q} & =-\left(\frac{c_{o} g}{\sqrt{N} M}\right) \sum_{i=1}^{2} \frac{b_{i}\left(a_{i} \sin \omega_{o} \tau-\omega_{o} \sin a_{i} \tau\right)}{a_{i} \omega_{o} \tau\left(a_{i}^{2}-\omega_{o}^{2}\right)}, \\
D_{p} & =-\left(\frac{c_{o} g}{\sqrt{N} M m}\right) \sum_{i=1}^{2} \frac{b_{i}\left[a_{i}^{2}\left(1-\cos \omega_{o} \tau\right)-\omega_{o}^{2}\left(1-\cos a_{i} \tau\right)\right]}{a_{i}^{2} \omega_{o}^{2} \tau\left(a_{i}^{2}-\omega_{o}^{2}\right)} .
\end{aligned}
$$

It is noted that $R$ given by Eqs. (72), (77), (79)-(83) is independent of $N$ because $\sqrt{N}$ factor in the first term of Eq. (72) is cancelled out by $1 / \sqrt{N}$ of $D_{q}$ in Eq. (82), and because $N$ factors in the third and fourth terms in Eq. (72) are cancelled out by $1 / N$ factors of $D_{q}^{2}$ and $D_{p}^{2}$ in Eqs. (82) and (83). This is the consequence of our choice of $c_{n}$ in Eq. (15): a different choice of the $N$ dependence of $c_{n}$ leads to $N$-dependent $R$. Furthermore $R$ is independent of $\beta$ because $\beta$ factor in the second term of Eq. (77) is cancelled out by $1 / \beta$ in Eq. (72).

Figure 3(a) shows the $\tau$ dependence of $\mu(=\langle W\rangle)$ for $c_{o}=0.0$ (solid curves), 0.5 (dashed curves) and 1.0 (chain curves). For $\tau \lesssim T_{o}(\simeq 6)$, we obtain $\langle W\rangle>\Delta F(=-0.5)$ signaling the occurrence of the irreversibility. At the same time, $\sigma\left(=\sqrt{\left\langle(W-\langle W\rangle)^{2}\right\rangle}\right)$ is rapidly increased for $\tau \lesssim T_{o}$, where fluctuation in $W$ much grows, as shown in Fig. 3(b). For $\tau=2 m \pi / \Omega(m=1,2, \cdot$.$) with c_{o}=0.0, \sigma$ vanishes [Eq. (93)]. Figure 3(c) will be explained shortly.

Figure 4 shows $3 D$ plots of WDF of $P(W)$ as functions of $W$ and $\tau$ for $c_{o}=0.0$ : result for $c_{o}=1.0$ is not so different from that of $c_{o}=0.0$ on first glance. With decreasing $\tau$, the center of $P(W)$ moves to zero and its width is considerably increased as Figs. 3(a) and 3(b) show. 


\section{Jarzynski equality}

In this subsection, we consider the JE given by Eq. (11). From Eqs. (11) and (75)-(77), the $\mathrm{JE}$ is satisfied if the relation given by

$$
R=\phi-\frac{\beta \sigma^{2}}{2}=\Delta F,
$$

holds. Here $\Delta F$ denotes the free energy difference between the two equilibrium systems with and without a force $g$ defined by $[9]$

$$
\Delta F=F(g)-F(0)=-\frac{1}{\beta} \ln \frac{Z_{S}(g)}{Z_{S}(0)},
$$

with

$$
Z_{S}(g)=\frac{\operatorname{Tr}\left\{e^{-\beta\left[H_{S}(g)+H_{B}+H_{I}\right]}\right\}}{\operatorname{Tr}\left\{e^{-\beta H_{B}}\right\}},
$$

where

$$
\begin{aligned}
H_{S}(g) & =\frac{P^{2}}{2 M}+\frac{M \Omega^{2}}{2}\left(Q-\frac{g}{M \Omega}\right)^{2}-\frac{g^{2}}{2 M \Omega}, \\
H_{B} & =\sum_{n=1}^{N}\left(\frac{p_{n}^{2}}{2 m}+\frac{m \omega_{n}^{2} q_{n}^{2}}{2}\right), \\
H_{B}+H_{I} & =\sum_{n=1}^{N}\left[\frac{p_{n}^{2}}{2 m}+\frac{m \omega_{n}^{2}}{2}\left(q_{n}-\frac{c_{n} Q}{m \omega_{n}^{2}}\right)^{2}\right],
\end{aligned}
$$

$Z_{S}(g)$ denoting the partition function of the system with $H_{S}(g)$ for a constant force of $f(t)=g$. By using Eqs. (85)-(89), we obtain

$$
Z_{S}(g)=\left(\frac{2 \pi}{\beta \Omega}\right) e^{\beta g^{2} / 2 M \Omega^{2}},
$$

yielding

$$
\Delta F=-\frac{g^{2}}{2 M \Omega^{2}},
$$

which is independent of the coupling $c_{o}$.

In what follows, we examine $\mu, \sigma^{2}$ and $R$ in the three limits of (1) no couplings $\left(c_{o}=0\right)$, (2) transient force $(\tau \rightarrow 0)$ and (3) quasi-static force $(\tau \rightarrow \infty)$.

(1) In the limit of $c_{o}=0$, we obtain from Eqs. (72) and (79)-(83),

$$
\begin{aligned}
\mu & =-\frac{g^{2}}{2 M \Omega^{2}}+\frac{g^{2}(1-\cos \Omega \tau)}{M \Omega^{4} \tau^{2}}, \\
\sigma^{2} & =\frac{2 g^{2}(1-\cos \Omega \tau)}{\beta M \Omega^{4} \tau^{2}},
\end{aligned}
$$


leading to

$$
R=-\frac{g^{2}}{2 M \Omega^{2}}=\Delta F
$$

where $\Delta F$ is given by Eq. (91).

(2) In the limit of $\tau \rightarrow 0$, Eqs. (72) and (79)-(83) lead to

$$
\begin{aligned}
\mu & =0 \\
\sigma^{2} & =\frac{g^{2}}{\beta M \Omega^{2}},
\end{aligned}
$$

where we employ the relations: $C_{Q}=-g$ and $C_{P}=D_{q}=D_{p}=0$. A substitution of Eq. (96) into Eq. (777) leads to

$$
R=-\frac{g^{2}}{2 M \Omega^{2}}=\Delta F
$$

(3) In limit of $\tau \rightarrow \infty$, we obtain

$$
\begin{aligned}
\mu & =-g^{2} \sum_{i=1}^{2} \frac{b_{i}}{2 M a_{i}^{2}}=-\frac{g^{2}}{2 M \Omega^{2}} \\
\sigma^{2} & =0
\end{aligned}
$$

employing the relations: $\sum_{i=1}^{2}\left(b_{i} / a_{i}^{2}\right)=1 / \Omega^{2}$ and $C_{Q}=C_{P}=D_{q}=D_{p}=0$. Equations (77) and (99) lead to

$$
R=-\frac{g^{2}}{2 M \Omega^{2}}=\Delta F
$$

Equations (94), (97), and (100) imply that the JE holds in the three limits of $(1) c_{o}=0,(2)$ $\tau \rightarrow 0$ and (3) $\tau \rightarrow \infty$.

Figure 3(c) shows that the JE is numerically verified for $10^{-1} \leq \tau \leq 10^{2}$ with $c_{o}=0.0$, 0.5 and 1.0. The JE is valid even when we adopt other sets of model parameters. It is surprising that complicated expressions of $\mu(=\phi)$ and $\sigma^{2}$ given by Eqs. (779) and (72), respectively, satisfy the JE given by Eq. (84). Although the validity of the JE is confirmed by numerical calculations, we have not succeeded in its analytical proof except for the three cases of $c_{o}=0, \tau \rightarrow 0$ and $\tau \rightarrow \infty$. 


\section{DISCUSSION}

\section{A. Canonical average over initial equilibrium state}

It should be stressed that the canonical average in Eq. (35) must be performed over the total Hamiltonian $H\left(=H_{S}+H_{B}+H_{I}\right)$ in the initial equilibrium state [61]. If the average in Eq. (35) is performed over the Hamiltonian of the uncoupled state $\left(H_{S}+H_{B}\right)$ in place of $H$, we obtain a wrong result. Figures 5(a) and (b) show $\bar{E}_{S}(t)\left(=\left\langle E_{S}(t)\right\rangle_{00}\right)$ with no forces $(f=0.0)$ and a ramp force of $\tau=100$, respectively, with $c_{o}=1$ when the average is performed over the initial uncoupled state of $\left(H_{S}+H_{B}\right)$,

$$
\langle O\rangle_{00} \equiv \frac{\operatorname{Tr}\left\{e^{-\beta\left[H_{S}(0)+H_{B}(0)\right]} O\right\}}{\operatorname{Tr} e^{-\beta\left[H_{S}(0)+H_{B}(0)\right]}},
$$

where $O$ stands for an operator. Results in Figs. 5(a) and (b) are quite different from the corresponding ones averaged over $H$ which have been shown in Figs. 2 (b) and (d). In particular, the irregular energy exchange between the system and bath occurs even when $f(t)=0.0$ in Fig. 5(a), while the initial serene state persists in Figs. 2(b). Figure 5(a) denotes the result of the case where the system-bath coupling is suddenly added at $t=0.0$ to the uncoupled system in equilibrium state at $t<0.0$. The perturbation of the added coupling induces the irregular energy exchange between the system and bath which does not dissipate for $t \geq 0.0$.

If the canonical average in Eq. (178) is performed over $H_{S}+H_{B}$, we obtain

$$
\left\langle e^{-\beta W_{0}}\right\rangle_{00}=e^{-\beta\left(\phi-\beta \sigma^{\prime 2} / 2\right)},
$$

with

$$
\sigma^{\prime 2}=\frac{1}{\beta}\left[\frac{C_{Q}^{2}}{M \Omega^{2}}+M C_{P}^{2}+\frac{N D_{q}^{2}}{m \omega_{o}^{2}}+m N D_{p}^{2}\right],
$$

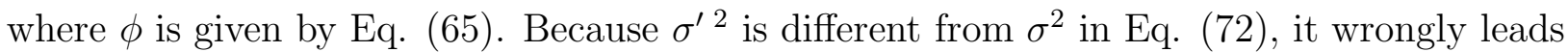
to a violation of the JE: $R=\phi-\beta \sigma^{2} / 2 \neq \phi-\beta \sigma^{2} / 2=\Delta F$. The related discussion will be given also in Sec. III.D. 


\section{B. A two-step ramp force}

Besides a ramp force given by Eq. (47), we have employed a two-step ramp force given by

$$
f(t)= \begin{cases}0 & \text { for } t<0, \\ g\left(\frac{h t}{\tau_{m}}\right) & \text { for } 0 \leq t<\tau_{m} \\ g\left[\frac{(1-h) t+\left(h \tau-\tau_{m}\right)}{\left(\tau-\tau_{m}\right)}\right] & \text { for } \tau_{m} \leq t<\tau, \\ g & \text { for } t \geq \tau\end{cases}
$$

where $h$ stands for a magnitude at a middle time of $\tau_{m}(<\tau)$. Figure 6 (a)and (b) show $\mu$ and $\sigma$, respectively, as a function of $\tau$ when a two-step ramp input $f(t)$ given by Eq. (104) with $g=1.0, h=1.5$ and $\tau_{m}=\tau / 2$ is applied $[f(t)$ is shown in the inset of Fig. 6(b)]. The input force $f(t)$ first linearly increases to $1.5 \mathrm{~g}$ at $t=\tau_{m}$ and then it linearly decreases to the final value of $g$ at $t \geq \tau$. The $\tau$ dependences of $\mu$ and $\sigma$ shown in Fig. 6 are rather different from those for a single-step ramp input shown in Fig. 3. In particular, magnitudes of $\mu$ and

$\sigma$ have resonance-like peaks at $\tau \sim T_{o}$. Nevertheless the JE holds also for the two-step ramp force.

\section{Baths with multiple $\omega$ and infinite $N$}

Our study in the preceding section has been made for the CL model with the single- $\omega$ bath, which may be extended to multiple- $\omega$ bath. The Green's function given by Eq. (12) may be generally expressed by

$$
\hat{G}(s)=\sum_{i=1}^{N+1} \frac{b_{i}}{\left(s^{2}+\tilde{\omega}_{i}^{2}\right)},
$$

where $\tilde{\omega}_{i}$ denotes the normal-mode frequency of the coupled system-plus-bath and $b_{i}$ is expressed in terms of the corresponding eigenfunction [62].

The inverse Laplace transformation leads to

$$
G(t)=\sum_{i=1}^{N+1} \frac{b_{i} \sin \tilde{\omega}_{i} t}{\tilde{\omega}_{i}} .
$$

The Green's function given by Eq. (105) or (106) has the same structure as that for the single- $\omega$ bath given by Eq. (18). Calculations of $Q(t), W_{0}$ and $P(W)$ may be formally 
performed in the same way as was made in Sec. II. Then properties of the CL model with finite- $N$ multiple- $\omega$ bath are essentially the same as those with single- $\omega$ bath.

On the other hand, in the limit of $N \rightarrow \infty$, the summation over $n$ in the Green's function of Eq. (12) is converted to integral over a continuous spectrum and it may be expressed by

$$
\hat{G}(s)=\left[s^{2}+\Omega^{2}+\frac{s^{2} c_{o}^{2}}{M m} \int \frac{D(\omega)}{w^{2}\left(s^{2}+\omega^{2}\right)} d \omega\right]^{-1},
$$

where $D(\omega)$ denotes the density of state,

$$
D(\omega)=\frac{1}{N} \sum_{n=1}^{N} \delta\left(\omega-\omega_{n}\right) .
$$

When we assume the Debye-type density of states: $D(\omega)=a \omega^{2}$ (a: constant), $\hat{G}(s)$ is given by

$$
\hat{G}(s)=\frac{1}{\left(s+c_{1}\right)\left(s+c_{2}\right)}
$$

with

$$
c_{1,2}= \pm i \sqrt{\Omega^{2}-\left(\frac{\pi a c_{o}^{2}}{4 M m}\right)^{2}}+\left(\frac{\pi a c_{o}^{2}}{4 M m}\right) .
$$

Because of real parts in $c_{1}$ and $c_{2}$, the inverse Laplace transformation of $\hat{G}(s)$ in Eq. (109) yields dissipative $G(t)$ which vanishes at $t \rightarrow \infty$. For dissipation it is necessary that the frequencies $\left\{\omega_{n}\right\}$ have a continuous spectrum in the limit of $N \rightarrow \infty$ [62]. With a discrete spectrum for finite $N$, however, the Green's function $G(t)$ in Eq. (12) is non-dissipative and not vanishing in the limit of $t \rightarrow \infty$.

\section{The generalized Langevin approach}

In the conventional approach to the CL model, we derive the Langevin equation given by

$$
M \ddot{Q}=-M \Omega^{2} Q-\int_{0}^{t} \gamma\left(t-t^{\prime}\right) \dot{Q}\left(t^{\prime}\right) d t^{\prime}+\zeta^{\prime}(t)+f(t),
$$

with

$$
\begin{aligned}
\zeta^{\prime}(t) & =\zeta(t)-\gamma(t) Q(0) \\
\gamma(t) & =\sum_{n=1}^{N}\left(\frac{c_{n}^{2}}{m \omega_{n}^{2}}\right) \cos \omega_{n} t \\
\zeta(t) & =\sum_{n=1}^{N} c_{n}\left[q_{n}(0) \cos \omega_{n} t+\left(\frac{p_{n}(0)}{m \omega_{n}}\right) \sin \omega_{n} t\right] .
\end{aligned}
$$


after obtaining a formal solution of $q_{n}(0)$ from Eq. (7) and substituting it into Eq. (6) [50, 51]. Equations (111)-(114) express the non-Markovian Langevin equation with colored noise.

When we adopt the single- $\omega$ bath given by Eq. (13), $\gamma(t)$ and $\zeta(t)$ are given by

$$
\begin{aligned}
\gamma(t) & =\left(\frac{c_{o}^{2}}{m \omega_{o}^{2}}\right) \cos \omega_{o} t, \\
\zeta(t) & =\frac{c_{o}}{\sqrt{N}}\left[\cos \omega_{o} t \sum_{n=1}^{N} q_{n 0}+\frac{\sin \omega_{o} t}{m \omega_{o}} \sum_{n=1}^{N} p_{n 0}\right] .
\end{aligned}
$$

By using the Laplace transformation yielding

$$
\begin{aligned}
& \hat{\gamma}(s)=\frac{c_{o}^{2} s}{m \omega_{o}^{2}\left(s^{2}+\omega_{o}^{2}\right)}, \\
& \hat{\zeta}(s)=\frac{c_{o}}{\sqrt{N}}\left[\frac{s}{s^{2}+\omega_{o}^{2}} \sum_{n=1}^{N} q_{n 0}+\frac{1}{m\left(s^{2}+\omega_{o}^{2}\right)} \sum_{n=1}^{N} p_{n 0}\right],
\end{aligned}
$$

we obtain an equation for $\hat{Q}(s)$ which is exactly the same as Eqs. (15) and (16).

It has been shown that the JE is satisfied in the non-Markovian Langevin model with colored noise (generalized Langevin model) [37-40], which is different from our Langevin equation given by Eqs. (111), (115) and (116) in the two points: (a) the second term of Eq. (112) includes an additional term of $-\gamma(t) Q(0)$ which is missing in the conventional generalized Langevin model, and (b) the memory kernel given by Eq. (115) is oscillating and non-dissipative while that in the generalized Langevin model is dissipative. In the literature (e.g. Ref. [65]), the additional term of $-\gamma(t) Q(0)$ is discarded and the fluctuation-dissipation relation is given by

$$
\left\langle\zeta(t) \zeta\left(t^{\prime}\right)\right\rangle_{00}=k_{B} T \gamma\left(t-t^{\prime}\right)
$$

which is derived from the equi-partition relations: $\left\langle q_{n 0} q_{\ell 0}\right\rangle_{00}=\left(k_{B} T / m \omega_{n}^{2}\right) \delta_{n \ell}$ and $\left\langle p_{n 0} p_{\ell 0}\right\rangle_{00}=\left(k_{B} T m\right) \delta_{n \ell}$, the bracket $\langle\cdot\rangle_{00}$ denoting the canonical average over the uncoupled initial state $H_{S}+H_{B}$ [Eq. (101) $]$. If the additional term is included, we obtain

$$
\left\langle\zeta^{\prime}(t) \zeta^{\prime}\left(t^{\prime}\right)\right\rangle_{00}=k_{B} T\left[\gamma\left(t-t^{\prime}\right)+\frac{\gamma(t) \gamma\left(t^{\prime}\right)}{M \Omega^{2}}\right]
$$

which is different from Eq. (119). It is noted, however, that when employing Eqs. (29)-(34) valid for equilibrium initial states of coupled Hamiltonian $H\left(=H_{S}+H_{B}+H_{I}\right)$, we obtain the desired fluctuation-dissipation relation,

$$
\left\langle\zeta^{\prime}(t) \zeta^{\prime}\left(t^{\prime}\right)\right\rangle_{0}=k_{B} T \gamma\left(t-t^{\prime}\right) .
$$


Then the ostensible inconsistency of the item (a) may be resolved. As for the item (b), the importance of the ergodicity is emphasized in Refs. [39, 40] from a study on the JE for the generalized Langevin model. Non-ergodic solutions of the non-dissipative generalized Langevin equation have been discussed in Refs. [62 64]. Our non-diffusive memory kernel yields non-ergodic solutions for the Langevin equation given by Eqs. (111), (115) and (116). It is noted that the JE holds in our calculation even if the condition of the ergodicity is not satisfied, in contrast with Refs. [39, 40].

Before closing Sec. III, it is necessary to mention that Ref. [29] has studied the validity condition of the JE for a general classical dynamical system with any time-dependent external force $f(t)$. It is shown in [29] that the JE holds for a classical system during a transition process in which the value of a parameter $f$ in the system Hamiltonian switches from $f_{0}$ to $f_{1}$ in time $\tau$, as long as the phase space extension of the system $\Omega_{0}^{e q}$ at the initial equilibrium phase space with $f=f_{0}$ is the same as the equilibrium phase space $\Omega_{1}^{e q}$ with $f=f_{1}$. It is noted that the general validity condition reported in Ref.[29] is satisfied for our system even though it is a non-ergodic one.

\section{CONCLUSION}

We have studied the response to an applied force of small open oscillator system described by the exactly solvable CL model with the non-dissipative single- $\omega$ bath. Although the model adopted in our study seems a pedagogical toy model, it is expected not to be unrealistic because non-dissipative properties are realized in small systems coupled to finite baths [47, 48]. We have obtained exact expressions for position, momentum and energy of the system whose canonical averages have been analytically performed over initial equilibrium states. Our calculations of system energy and work have shown the following:

(i) the energy of the system strongly coupled to finite bath is fluctuating but non-dissipative in general, and

(ii) the JE is valid in non-dissipative non-ergodic systems.

The item (i) supports direct simulations for open systems coupled to finite baths [47, 48] although it is contrast to the result showing the dissipation for $N \gtrsim 10-20$ [58]. The item (ii) is consistent with Jarzynski's proof for an arbitrary classical open systems [9]. Our study is complementary to the previous studies for dissipative oscillator systems with the 
use of the Markovian [20 23] and non-Markovian Langevin models [37-40], Fokker-Planck equation [41] and Hamiltonian models [42-46].

Although the items (i) and (ii) hold for open systems described by the CL [50, 51] and Ford-Kac models [65], it is not certain whether they are valid for any non-dissipative nonergodic open system. In this respect, it would be interesting to examine a work in the $\left(N_{S}+N_{B}\right)$ model for a classical $N_{S}$-body system coupled to an $N_{B}$-body bath [48]. The $\left(N_{S}+N_{B}\right)$ model clarifies some interesting issues such as the $N_{S}$-dependent non-Gaussian energy distribution of the system [48] which has been not realized in previous studies for CL-type models with $N_{S}=1$ [50, 51, 65]. Such a calculation is in progress and will be reported in a separate paper.

\section{Acknowledgments}

The author expresses his sincere thanks to Prof. Peter Talkner and Prof. Juyeon Yi for pointing out a mistake in the first version of the manuscript. This work is partly supported by a Grant-in-Aid for Scientific Research from Ministry of Education, Culture, Sports, Science and Technology of Japan.

[1] C. Bustamante, J. Liphardt, and F. Ritort, Phys. Today 58, 4 (2005).

[2] F. Ritort, Nonequilibrium fluctuations in small systems: From physics to biology, in Advance in Chemical Physics, vol. 137, Ed. S. A. Rice (J. Wiley \& Sons, Inc., 2008).

[3] S. Ciliberto, S. Joubaud, A. Petrosyan, arXiv:1009.3362.

[4] C. Jarzynski, Phys. Rev. Lett. 78, 2690 (1997).

[5] D. J. Evans, E. G. D. Cohen, and G. P. Morriss, Phys. Rev. Lett. 71, 2401 (1993); D. J. Evans and D. J. Searles, Phys. Rev. E 50, 1645 (1994).

[6] O. Narayan and A. Dhar, J. Phys. A 37, 63 (2004).

[7] G. E. Crooks, Phys. Rev. E 60, 2721 (1999).

[8] C. Jarzynski, Phys. Rev. E 56, 5018 (1997).

[9] C. Jarzynski, J. Stat. Mech.: Theor. Exp. P09005 (2004).

[10] J. Kurchan, arXiv:cond-mat/0007360. 
[11] H. Tasaki, arXiv:cond-mat/0009244v2.

[12] S. Mukamel, Phys. Rev. Lett. 90, 170604 (2003).

[13] W. De Roeck and C. Maes, Phys. Rev. E 69, 026115 (2004).

[14] V. Chernyak and S. Mukamel, Phys. Rev. Lett. 93, 048302 (2004).

[15] T. Monnai, Phys. Rev. E 72, 027102 (2005).

[16] P. Talkner, E. Lutz and P. Hänggi, Phys. Rev. E 75, 050102R (2007).

[17] M. Campisi, P. Talkner, and P. Hänggi, Phys. Rev. Lett. 102, 210401 (2009).

[18] J. Liphardt, S. Dumont, S. Smith, I. Tinoco, C. Bustamante, Science 296, 1833 (2002).

[19] G. M. Wang, J. C. Reid, D. M. Carberry, D. R. M. Williams, E. M. Sevick, and Denis J. Evans, Phys. Rev. E 71, 046142 (2005).

[20] F. Douarche, S. Ciliberto, A. Petrosyan, and I. Rabbiossi, Europhys. Lett. 70, 593 (2005).

[21] F. Douarche, S. Joubaud, N. B. Garnier, A. Petrosyan, and S. Ciliberto, Phys. Rev. Lett. 97, $140603(2006)$.

[22] S. Joubaud, N. B. Garnier, F. Douarche, A. Petrosyan, and S. Ciliberto, C. R. Physique 8, $518(2007)$.

[23] S. Joubaud, N. B. Garnier, S. Ciliberto, J. Stat. Mech., P09018 (2007).

[24] E. G. D. Cohen and D. Mauzerall, J. Stat. Mech., P07006 (2004).

[25] D. H. E. Gross, arXiv:cond-mat/0508721.

[26] J. Sung, arXiv:cond-mat/0506214.

[27] J. Sung, arXiv: cond-mat/0510119.

[28] M. Bier, arXiv:cond-mat/0510270.

[29] J. Sung, arXiv: cond-mat/0512250.

[30] R. C. Lua and A. Y. Grosberg, J. Phys. Chem. B 109, 6805 (2005).

[31] I. Bena, C. van den Broeck and R. Kawai, Europhys. Lett. 71, 879 (2005).

[32] Z. C. Tu and Z. Zhou, Commun. Theor. Phys. 46, 886 (2006).

[33] S. Pressé and R. Silbey, J. Chem. Phys. 124, 054117 (2006).

[34] J. Sung, Phys. Rev. E 76, 012101 (2007).

[35] J. Sung, Phys. Rev. E 77, 042101 (2008).

[36] W. A. M. Morgado and D. O. Soares-Pinto, Phys. Rev. E 82, 021112 (2010).

[37] F. Zamponi, F. Bonetto, L. F. Cugliandolo and J. Kurchan, J. Stat. Mech. P09013 (2005).

[38] T. Mai and A. Dhar, Phys. Rev. E 75, 061101 (2007). 
[39] T. Speck and U. Seifert, arXiv:0709.2236, J. Stat. Mech. L09002 (2007).

[40] T. Ohkuma and T. Ohta, J. Stat. Mech. P10010 (2007).

[41] S. Chaudhury, D. Chatterjee and B. J Cherayil, J. Stat. Mech. P10006 (2008).

[42] A. Dhar, Phys. Rev. E 71, 036126 (2005).

[43] C. Jarzynski, Comptes Rendus Physique 8, 495 (2007).

[44] C. Jarzynski, Eur. Phys. J. B. 64, 331 (2008).

[45] R. Chakrabarti, arXiv: 0802.0268.

[46] H. Hijar and J. M. O. de Zárate, Eur. J. Phys. 31, 1097 (2010).

[47] A. V. Plyukhin and J. Schofield, Phys. Rev. E 64, 041103 (2001).

[48] H. Hasegawa, Phys. Rev. E 83, 021104 (2011).

[49] R. Zwanzig, Nonequilibrium Statistical Mechanics, (Oxford University Press, Oxford, 2001).

[50] A. O. Caldeira and A. J. Leggett, Phys. Rev. Lett. 46, 211 (1981).

[51] A. O. Caldeira and A. J. Leggett, Ann. Phys. 149, 374 (1983).

[52] P. S. Riseborough, P. Hänggi, and U. Weiss, Phys. Rev. A 31, 471 (1985).

[53] In the CL model we assume $c_{n}=c_{o} / \sqrt{N}$ because the kernel $\gamma(t)$ includes the $c_{n}^{2}$ term: $\gamma(t)=\sum_{n=1}^{N} c_{n}^{2}\left(\cos \omega_{n} t / m \omega_{n}^{2}\right)[$ Eq. (113) $]$ which becomes $\gamma(t)=\int D(\omega)\left(\cos \omega t / m \omega^{2}\right) d \omega$ in the limit of $N \rightarrow \infty, D(\omega)$ standing for the density of states.

[54] Two methods have been proposed for calculating the system energy in the system-plus-bath [55]: $E_{S}^{(1)}=\operatorname{Tr}\left\{e^{-\beta H} H_{S}\right\} / \operatorname{Tr} e^{-\beta H}$ and $E_{S}^{(2)}=-\partial \ln Z_{S} / \partial \beta$ with the reduced partition function, $Z_{S}=\operatorname{Tr} e^{-\beta H} / \operatorname{Tr}_{B} e^{-\beta H_{B}}$ where $\operatorname{Tr}_{B}$ stands for the partial trace over the bath. We obtain $E_{S}^{(1)}=E_{S}^{(2)}$ for the classical CL model [55 57 ].

[55] P. Hänggi, G-L. Ingold and P. Talkner, New Journal of Physics 10, 115008 (2008).

[56] G-L. Ingold, P. Hänggi, and P. Talkner, Phys. Rev. E 79, 061105 (2009).

[57] M. F. Gelin and M. Thoss, Phys. Rev. E 79, 051121 (2009).

[58] J. Rosa and M. W. Beims, Phys. Rev. E 78, 031126 (2008).

[59] S. T. Smith and R. Onofrio, Eur. Phys. J. B 61, 271 (2008).

[60] Q. Wei, S. T. Smith, and R. Onofrio, Phys. Rev. E 79, 031128 (2009).

[61] J. Yi and P. Talkner, private communications.

[62] A. Dhar and K. Wagh, Euro. Phys. Lett. 79, 60003 (2007).

[63] J-D. Bao, P. Hänggi, and Y-Z. Zhuo, Phys, Rev. E 72, 061107 (2005).

[64] A.V. Plyukhin, arXiv:1101.4550. 
[65] G. W. Ford and M. Kac, J. Stat. Phys. 46, 803 (1987). 
FIG. 1: (Color online) The time dependence of the averaged position $\bar{Q}$ (solid curves) and momentum $\bar{P}$ (dashed curves); (a) $\tau=100.0, c_{o}=0.0$, (b) $\tau=100.0, c_{o}=1.0$, (c) $\tau=10.0, c_{o}=0.0$, (d) $\tau=10.0, c_{o}=1.0$, (e) $\tau=5.0, c_{o}=0.0$, (f) $\tau=5.0, c_{o}=1.0,(\mathrm{~g}) \tau=0.0, c_{o}=0.0$, and (h) $\tau=0.0, c_{o}=1.0$.

FIG. 2: (Color online) The time dependence of the averaged system energy $\bar{E}_{S}$; for no forces $(f=0)$ with (a) $c_{o}=0.0,(\mathrm{~b}) c_{o}=1.0$; for the ramp forces with (c) $\tau=100.0, c_{o}=0.0,(\mathrm{~d})$ $\tau=100.0, c_{o}=1.0,(\mathrm{e}) \tau=10.0, c_{o}=0.0,(\mathrm{f}) \tau=10.0, c_{o}=1.0,(\mathrm{~g}) \tau=5.0, c_{o}=0.0,(\mathrm{~h}) \tau=5.0$, $c_{o}=1.0,(\mathrm{i}) \tau=0.0, c_{o}=0.0$, and $(\mathrm{j}) \tau=0.0, c_{o}=1.0$.

FIG. 4: (Color online) 3D plots of $P(W)$ as functions of $W$ and $\tau$ for $c_{o}=0.0$, the ordinate of (b) being enlarged compared to that of (a).

FIG. 5: (Color online) The time dependence of $\bar{E}_{S}(t)$ with (a) no forces $(f=0)$ and (b) a ramp force of $\tau=100.0$ with $c_{o}=1.0$ when the average is performed over initial uncoupled state of $H_{S}+H_{B}$ : (a) and (b) should be compared to Figs. 2(b) and (d), respectively, which are averaged over the initial coupled state of $H_{S}+H_{B}+H_{I}$ (see text).

FIG. 3: (Color online) The $\tau$ dependence of (a) $\mu(=\langle W\rangle),(\mathrm{b}) \sigma\left(=\sqrt{\left\langle(W-\langle W\rangle)^{2}\right\rangle}\right)$ and (c) $R$ $\left(=-\beta^{-1} \ln \left\langle e^{-\beta W}\right\rangle\right)$ for $c_{o}=0.0$ (solid curves), 0.5 (dashed curves) and 1.0 (chain curves); arrows along the right ordinates in (a) and (c) express $\Delta F(=-0.5)$. In (c) $R=\Delta F$ for $c_{o}=0.0,0.5$ and 1.0 . 
FIG. 6: (Color online) The $\tau$ dependence of (a) $\mu(=\langle W\rangle)$ and (b) $\sigma\left(=\sqrt{\left\langle(W-\langle W\rangle)^{2}\right\rangle}\right)$ for a two-step ramp force $f(t)$ given by Eq. (104) with $g=1.0, h=1.5$ and $\tau_{m}=\tau / 2$ [see the inset of (b)] with $c_{o}=0.0$ (solid curves), 0.5 (dashed curves) and 1.0 (chain curves); an arrow along the right ordinate in (a) expresses $\Delta F(=-0.5)$. 
This figure "fig1.jpg" is available in "jpg" format from: http://arxiv.org/ps/1102.1715v3 
This figure "fig2.jpg" is available in "jpg" format from: http://arxiv.org/ps/1102.1715v3 
This figure "fig3.jpg" is available in "jpg" format from: http://arxiv.org/ps/1102.1715v3 
This figure "fig4.jpg" is available in "jpg" format from: http://arxiv.org/ps/1102.1715v3 
This figure "fig5.jpg" is available in "jpg" format from: http://arxiv.org/ps/1102.1715v3 
This figure "fig6.jpg" is available in "jpg" format from: http://arxiv.org/ps/1102.1715v3 